\title{
Noise-free stochastic resonance at an interior crisis
}

\author{
Thomas Jüngling* and Hartmut Benner \\ Institute for Solid State Physics, Darmstadt University of Technology, Hochschulstrasse 6, 64289 Darmstadt, Germany \\ Thomas Stemler \\ School of Mathematics and Statistics, University of Western Australia, 35 Stirling Highway, Crawley Western Australia 6009, Australia \\ Wolfram Just \\ School of Mathematical Sciences, Queen Mary/University of London, Mile End Road, London E1 4NS, United Kingdom
}

(Received 21 December 2007; published 24 March 2008)

\begin{abstract}
We report on the observation of noise-free stochastic resonance in an externally driven diode resonator close to an interior crisis. At sufficiently high excitation amplitudes the diode resonator shows a strange attractor which after the collision with an unstable period-three orbit exhibits crisis-induced intermittency. In the intermittency regime the system jumps between the previously stable chaotic attractor and the phase space region which has been made accessible by the crisis. This random process can be used to amplify a weak periodic signal through the mechanism of stochastic resonance. In contrast to conventional stochastic resonance no external noise is needed. The chaotic intrinsic dynamics plays the role of the stochastic forcing. Our data obtained from the diode resonator are compared with numerical simulations of the logistic map where a similar crisis-induced intermittency is observed.
\end{abstract}

DOI: 10.1103/PhysRevE.77.036216

PACS number(s): 05.45.Tp, 05.40.-a

\section{INTRODUCTION}

Stochastic resonance (SR), originally found for the overdamped motion of a particle in a double well potential [1], can nowadays be rated as a paradigm of nonlinear dynamics which occurs in various nonlinear systems driven by noise and some weak periodic input signal [2]. In the output of such systems the modulation frequency of the periodic drive is well pronounced at a distinguished nonzero noise intensity while at lower and higher noise intensities the periodic part tends to zero. This counterintuitive effect of high coherence at an optimal noise intensity is the characteristic feature of SR and is found in different types of systems, such as bistable, reset fire, threshold, and dynamical systems, from various fields of science; see, e.g., Refs. [3-7].

It is well known that chaotic systems exhibiting intermittency can also amplify a weak signal via SR [8-10]. Here the slow intermittent jump dynamics can be synchronized with the periodic modulation while the fast chaotic dynamics acts as a source of randomness. This effect is called noise-free stochastic resonance to underline that no external noise is needed. Such a phenomenon was studied in detail for the cases of Pomeau-Manneville intermittency [11], on-off intermittency [12-15], and crisis-induced intermittency at a merging crisis $[8,16,17]$.

In this paper we consider noise-free SR by experimental means at an interior crisis. Our experimental setup consists of a diode resonator where an interior crisis, arising from a period-three window, is observed. We compare our experimental data with numerical results of the logistic map. In both the real and the model systems, the two states of chaotic motion are quite different. Thus our systems show a pro-

\footnotetext{
*Thomas.Juengling@physik.tu-darmstadt.de
}

nounced asymmetry. For stochastic systems such an asymmetry levels off the main peak of amplification and shifts it to higher noise intensities [18].

The paper is organized as follows. In the next section we present the experimental setup and the basic bifurcation diagram. Section III is devoted to techniques - taking the logistic map as an example- - how to distinguish the two chaotic phases occurring at the crisis. These techniques allow us to determine the mean residence times which play a key role for understanding the SR effects reported in Sec. IV. We conclude with some remarks on the universality of the observed noise-free SR phenomena.

\section{EXPERIMENTAL SETUP}

The diode resonator shown in Fig. 1 is a simple and robust nonautonomous electronic circuit showing chaos at sufficiently strong excitation. We choose the amplitude of the sinusoidal drive $U_{0}$ as the control parameter keeping the fre-

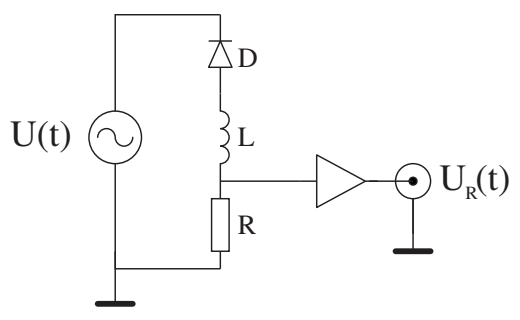

FIG. 1. Diode resonator circuit consisting of a $1 N 4006$ diode, an inductor $L=1 \mathrm{mH}$, a resistor $R=51 \Omega$, and a sinusoidal drive $U(t)$ $=U_{0} \sin (\Omega t)$ with amplitude $U_{0}=0-6 \mathrm{~V}$ and frequency $\Omega / 2 \pi$ $=1 \mathrm{MHz}$. Additional components for shielding and extracting the signal which were implemented to minimize noise and cross-talk are not shown. 


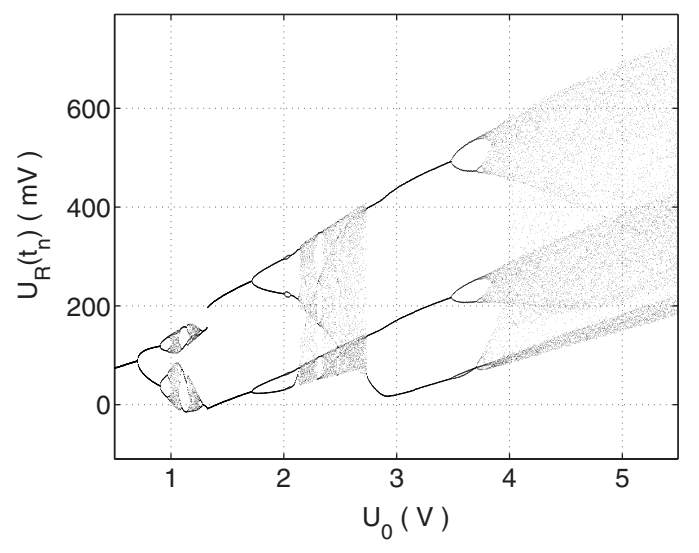

FIG. 2. Bifurcation diagram of the diode resonator constructed from the maxima of the output signal $U_{R}(t)$ at discrete times $t_{n}$.

quency $\Omega$ constant, and record the time series of the output signal $U_{R}(t)$. Plotting the maxima of $U_{R}(t)$ as a function of the control parameter $U_{0}$ the bifurcation diagram, Fig. 2, was obtained. The oscillator follows a period-doubling cascade ending up in chaos. The chaotic regime is riddled with periodic windows, each of them connected either with an additional period doubling sequence or a discontinuous transition.

For our analysis we focus on the period-three window which occurs in the range $U_{0}=2.7-4.0 \mathrm{~V}$. At $U_{0}=2.7 \mathrm{~V}$ the window opens in a saddle node bifurcation where a pair of stable and unstable period-three orbits is generated. At $U_{0}$ $=3.5 \mathrm{~V}$ the stable orbit starts bifurcating in a period doubling sequence which results in a period-three chaotic attractor for $U_{0}>3.8 \mathrm{~V}$. For later reference we will label such a state as "Ch1". At a critical value, $U_{c}=4.0 \mathrm{~V}$, the period-three chaotic attractor is destroyed in a crisis and a mixing chaotic state occurs. The chaotic attractor now fills the gaps between the bands, and we will label such gaps by "Ch2". In Fig. 3 the band attractor of period three exemplifies the effect of such an interior crisis. This type of crisis which closes the periodic window results in chaos-chaos intermittency where the system alternates between the different types of chaotic motion, labeled "Ch1" and "Ch2". Figure 4 shows a typical time series $U_{R}(t)$ from the intermittency regime. The times $\tau_{1}$ and $\tau_{2}$ a trajectory spends in each state were found to be larger than the time scale at which correlations of the chaotic motion decay. Thus, as usual, one can treat this type of intermittency similar to a dichotomous random process.

\section{IDENTIFICATION OF STATES}

A typical "burst" event (Ch2) as displayed in Fig. 4 shows up as a small slump of amplitude and an interrupt of the still present periodicity which is characteristic for the state "Ch1." However, a clear characteriazation of the intermittent states directly from the time series $U_{R}(t)$ turns out to be rather difficult, in particular, when trying to accomplish this procedure automatically. Different parts of the attractor corresponding to the phases $\mathrm{Ch} 1$ and $\mathrm{Ch} 2$ are convoluted in such a way that a separation by a simple threshold condition
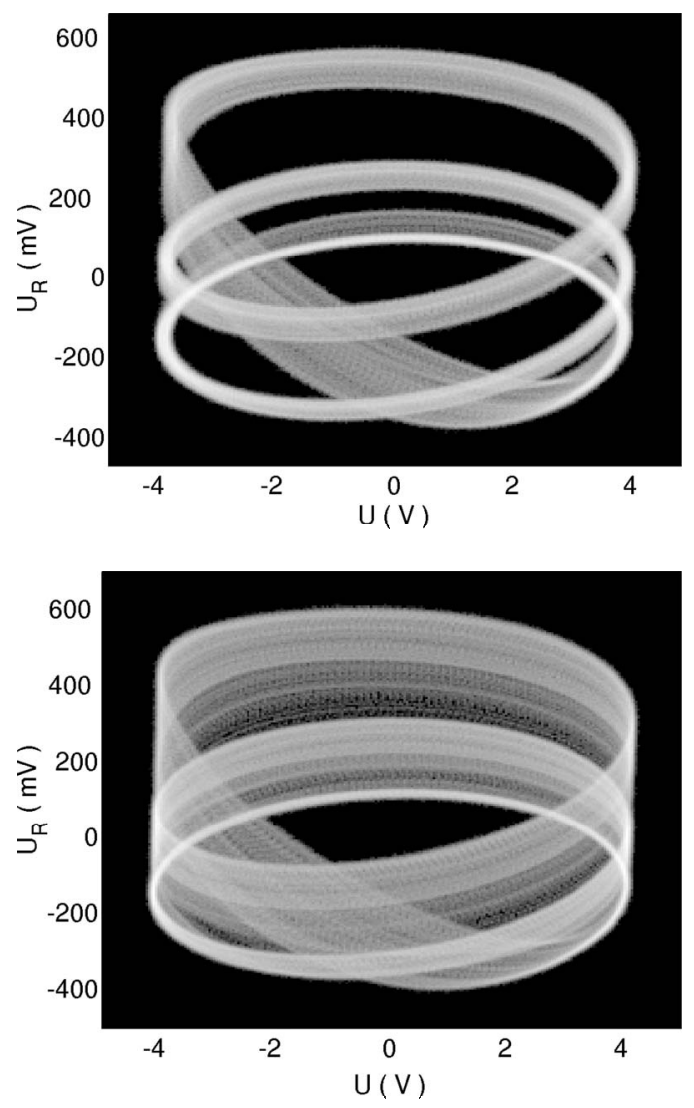

FIG. 3. Density plots showing the projection of the attractor onto the $U-U_{R}$ plane as a screen shot from an analog oscilloscope. Top: Driving amplitude $U_{0}<U_{c}=4.0 \mathrm{~V}$. Bottom: $U_{0}>U_{c}$.

is not feasible. A criterion can be derived by considering details of the jump processes in terms of a return map.

First, we illustrate this idea at an interior crisis of the logistic map

$$
x_{n+1}=r x_{n}\left(1-x_{n}\right) \equiv M_{r}\left(x_{n}\right) .
$$

Figure 5 shows a small part of the bifurcation diagram containing the period-three window. A saddle-node pair of

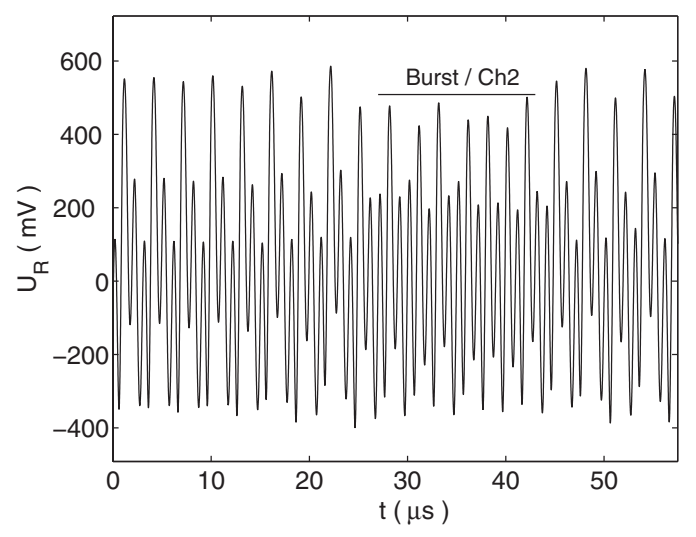

FIG. 4. Typical time trace in the intermittent regime showing a single burst. 


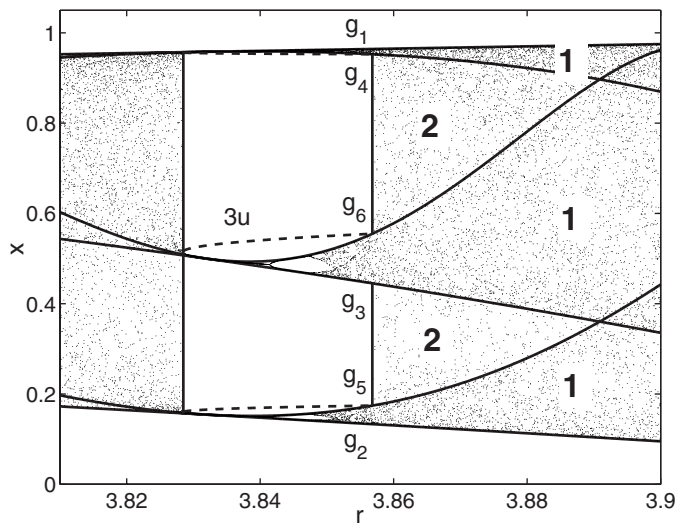

FIG. 5. Skeleton of the logistic map in a neighborhood of the period three window. Solid lines: first six iterates $g_{n}(r)$ of the critical point; broken line: unstable period-three orbit. Labels refer to the phases "Ch1" and "Ch2".

period-three orbits is generated at $r=1+\sqrt{8} \approx 3.828$ (see Ref. [19]). Via a cascade of period doublings a period-three chaotic state is generated. The unstable period-three orbit created in the saddle-node bifurcation determines the basin boundary of chaotic motion with different phases and thus prevents the period-three chaotic attractor to fill the gaps between the bands. At $r_{c} \approx 3.8568$ the unstable periodic orbit collides with the boundary of the chaotic attractor causing an interior crisis and resulting in a single band mixing chaotic state. To distinguish the former chaotic attractor, the "laminar state" Ch1, from the "burst" dynamics $(\mathrm{Ch} 2)$ we resort to the skeleton of the logistic map. The first six images of the critical point $x=1 / 2, g_{n}(r)=M_{r}^{n}(1 / 2), n=1,2, \ldots, 6$ determine the boundary of the period-three chaotic state for $r \lessgtr r_{c}$, since the critical point is contained in one of the chaotic bands and the three intervals are mapped onto each other [20]. Beyond the crisis, for $r>r_{c}$, the borderline functions $g_{n}(r)$ are smooth continuations of the precritical attractor defining the Ch1 regime. For maps with critical points these borderlines can be observed as the most significant structures in the invariant density. Such structures arise from folding the phase space during one iteration step. Because the derivative vanishes at the critical point a high density is created at the next image points $g_{n}$ while the density decays on further iteration due to the stretching of the chaotic map. Symbolic dynamics tells us that the three intervals $\left[g_{2}, g_{5}\right],\left[g_{3}, g_{6}\right]$, and $\left[g_{4}, g_{1}\right]$ constitute the period-three chaotic state for $r<r_{c}$ while the gaps are determined by $\left(g_{5}, g_{3}\right)$ and $\left(g_{6}, g_{3}\right)$. Thus, even beyond the crisis we can define the two different chaotic phases $\mathrm{Ch} 1$ and $\mathrm{Ch} 2$ by the symbolic variable

$$
\sigma_{n}=\left\{\begin{array}{c}
+1 \quad \text { if } x_{n} \in\left(g_{5}, g_{3}\right) \cup\left(g_{6}, g_{4}\right), \\
-1 \quad \text { if } x_{n} \in\left[g_{2}, g_{5}\right] \cup\left[g_{3}, g_{6}\right] \cup\left[g_{4}, g_{1}\right] .
\end{array}\right.
$$

Such a symbolic representation constitutes the basis for the further data analysis.

We now turn to the identification of the different dynamical regimes in our experimental system. The diode resonator follows a bifurcation scenario very similar to that of the logistic map. An appropriate return map $u_{n} \mapsto u_{n+1}$ can be con-

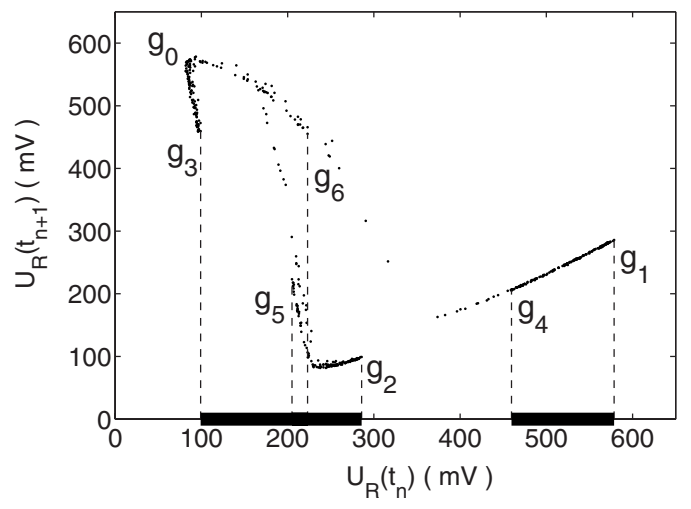

FIG. 6. Return map constructed from the local maxima of the experimental time series $U_{R}(t)$ close to the crisis. Labels refer to the critical point and its images. The rightmost branch of the Ch1 phase remains well separated, while the other branches overlap with each other and with parts of the $\mathrm{Ch} 2$ phase.

structed from the local maxima $u_{n} \equiv U_{R}\left(t_{n}\right)$ of the time series. But the resulting structure, shown in Fig. 6, is far more complex than the logistic map since the experimental data represent a one-dimensional projection of a higher-dimensional chaotic system. In contrast to the logistic map the projection of the three branches of the experimental band attractor onto the horizontal axis are no longer separated but partly overlap. Even more, the Ch1 and Ch2 phases are highly intermingled and more sophisticated tools are required to separate both states.

Despite these difficulties the main ideas developed in the context of the logistic map can be used here as well to separate the two different regimes of the intermittent dynamics. Again, the maximal value of the amplitude

$$
g_{1}=\max _{n}\left\{u_{n}\right\}=\max _{t}\left\{U_{R}(t)\right\}
$$

is of crucial importance. If our dynamical system were the logistic map then one of the preimages would be the critical point $g_{0}$ causing the folding of the phase space. Similar topological considerations apply to our experimental results as well (see Fig. 6), although we do not have to resort to a one-dimensional map. The following five recursive images $g_{2}, \ldots, g_{6}$ are again boundary points for each of the three branches. Below the crisis, for $U_{0}<U_{c}$, one can clearly observe that the different branches of the attractor Ch1 are mapped onto each other and visited in a well-defined sequence [see Fig. 7(a)]. Beyond the crisis, for $U_{0}>U_{c}$, the combined folding and stretching process maps some of the images outside the starting branch [see Fig. 7(b)], generating the $\mathrm{Ch} 2$ phase of the dynamics. The return map is neither a single valued function nor are the different branches of the phases well separated when projected onto the horizontal axis. Thus no simple threshold condition on the amplitudes $u_{n}$, such as Eq. (2), can be applied. Taking into account how the different branches of our return map are mapped onto each other we can develop a simple recipe to decompose the time series. The points $g_{1}$ and $g_{4}$ can be used to isolate the nonoverlapping first branch of the Ch1 phase. If $u_{n}$ 


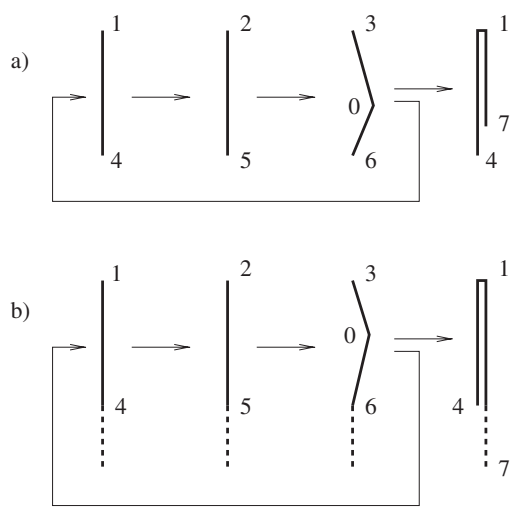

FIG. 7. Diagrammatic view of the folding and stretching process of a chaotic three-band attractor. (a) Below the crisis. Labels refer to the images of the "critical point" $g_{0}$; solid lines visualize the three bands which are mapped onto each other. (b) Beyond the crisis. Dashed lines indicate those parts which are not mapped onto each other any more.

$\in\left[g_{4}, g_{1}\right]$ we assign the symbol $\sigma_{n}=-1$ to this event and to the two following iterates. Those data points which after three iterations do no longer fit into the branch cycle Fig. 7 are attributed to the phase $\mathrm{Ch} 2$ by assigning a symbolic coordinate with positive value. The same applies to their followers as long as there is no event $u_{n} \in\left[g_{4}, g_{1}\right]$ detected.

By exploiting this technique the experimental time series can be converted into a symbolic sequence, as was already demonstrated for the logistic map. We use this sequence for further analysis, e.g., for the evaluation of the mean residence times $\left\langle\tau_{1}\right\rangle$ and $\left\langle\tau_{2}\right\rangle$ of the two different phases Ch1 and $\mathrm{Ch} 2$, respectively. The dependence of the mean residence time on the control parameter $U_{0}$ is shown in Fig. 8. The mean laminar residence time $\left\langle\tau_{1}\right\rangle$ follows a power law [21-25]. The exponent is related to the geometric properties of the attractor. The mean burst residence time $\left\langle\tau_{2}\right\rangle$ tends to a finite limit when approaching the crisis. Analytic expressions for scaling exponents have been derived in Ref. [26] for one-dimensional maps.

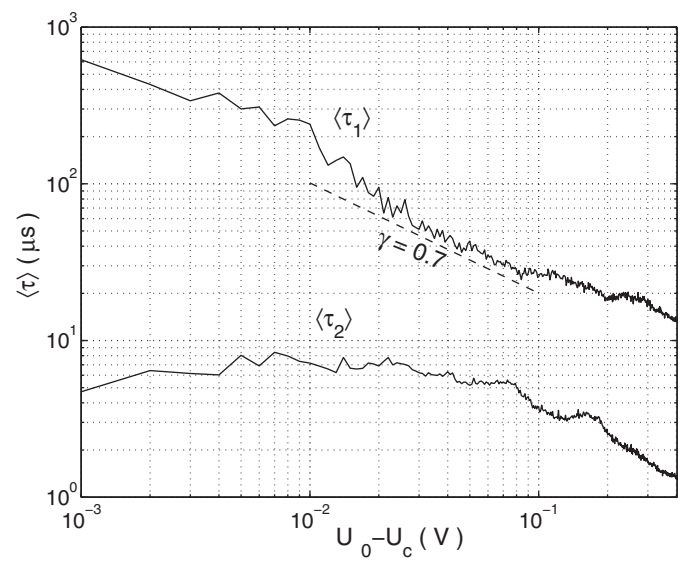

FIG. 8. Mean residence times of the diode resonator evaluated from the symbolic time series. $\left\langle\tau_{1}\right\rangle$ follows a power law with a scaling exponent $\gamma$ of 0.7 as predicted in Ref. [21].

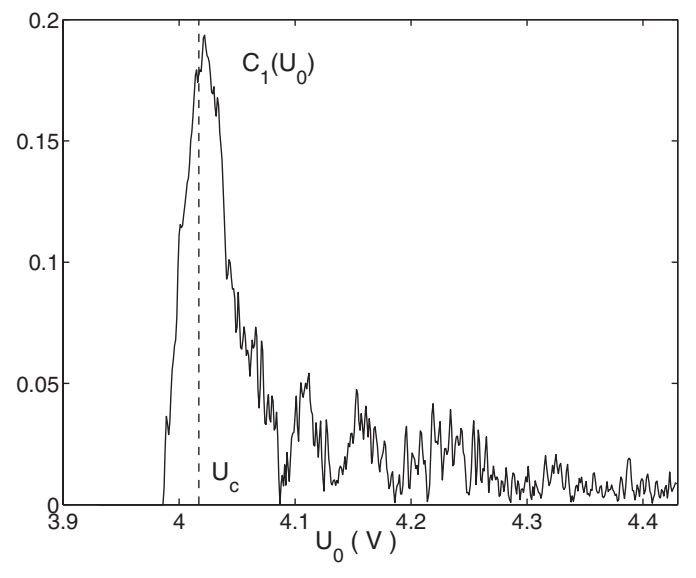

FIG. 9. Noise-free stochastic resonance of the diode resonator evaluated in terms of the normalized cross correlation function between the symbolic time series and the modulation signal (5).

\section{NOISE-FREE STOCHASTIC RESONANCE}

For studying the phenomenon of noise-free stochastic resonance we applied a slow amplitude modulation to the fast external excitation of the diode resonator. The constant drive amplitude $U_{0}$ was replaced by

$$
\widetilde{U}_{0}(t)=U_{0}\left[1+m \sin \left(\omega_{m} t\right)\right], \omega_{m} \ll \Omega .
$$

Here we choose $\omega_{m}=10 \mathrm{kHz}$ and $m=0.01$ so that for $U_{0}$ slightly above the critical value $U_{c}$ the modulation frequency is of the same order of magnitude as the jump rate between the two different states $\mathrm{Ch} 1$ and $\mathrm{Ch} 2$. The symbolic time series can be compared with the modulation signal by means of the cross correlation function $\left\langle\sigma_{n} \widetilde{U}_{0}\left(t_{n}+t\right)\right\rangle$. If we account for the nonvanishing time average of the output signal by considering the fluctuation $\delta \sigma_{n}=\sigma_{n}-\left\langle\sigma_{n}\right\rangle$, then the amplitude of the normalized cross correlation function is given by

$$
C_{1}=\frac{\max _{t}\left|\left\langle\delta \sigma_{n} \sin \left[\omega_{m}\left(t_{n}+t\right)\right]\right\rangle\right|}{\sqrt{\left\langle\left(\delta \sigma_{n}\right)^{2}\right\rangle / 2}} .
$$

Figure 9 shows the amplitude in dependence on the bifurcation parameter $U_{0}$. Well below the onset of the crisis $U_{0}$ $\ll U_{c}$ the correlation amplitude $C_{1}$ is small because the slow periodic drive does not induce phase slips among the periodthree chaotic motion. Very close to the crisis $U_{0} \approx U_{c}$ the correlation function shows a dramatic increase and reaches a sharp maximum which is a characteristic feature of noisefree SR. This main peak arises from the change of the burst rate from zero to a finite value when the control parameter crosses $U_{c}$ (see Refs. [16,27]). In addition, we observe several smaller peaks of comparable width for larger values of the driving amplitude. Such maxima correspond to several nonmonotonic bumps of the jump rate as a function of $U_{0}$ which were already visible in our data for the mean residence time $\left\langle\tau_{1}\right\rangle$; see Fig. 8 [24,25].

In order to show that the observed noise-free SR represents a universal property of an interior crises we also considered the logistic map introduced above as a simple model 


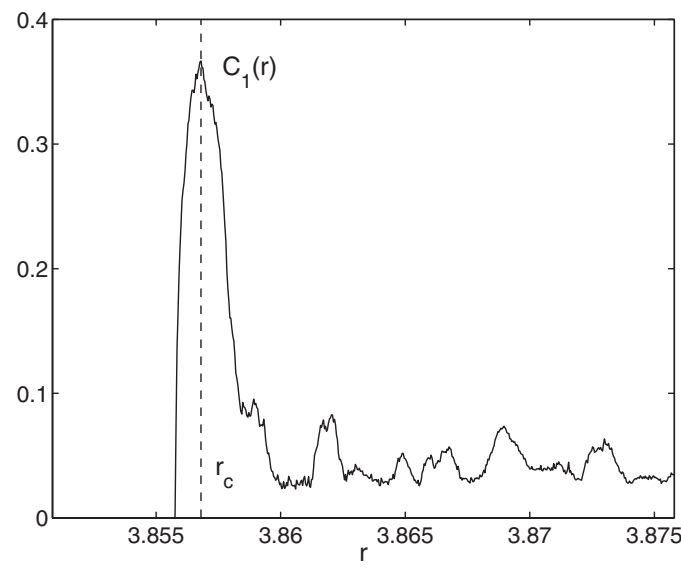

FIG. 10. Noise-free stochastic resonance of the logistic map. Normalized cross correlation function $C_{1}$ between the output signal $\sigma_{n}$ and the modulation $\tilde{r}_{n}$. The period of the modulation signal is $T_{m}=100$ iteration steps, which is comparable to the parameters $\omega / \omega_{m}$ chosen for the diode resonator.

which also exhibits interior crises. We applied a periodic parameter modulation

$$
\widetilde{r}_{n}=r\left[1+m \sin \left(\omega_{m} n\right)\right]
$$

to the one-dimensional map $x_{n+1}=\widetilde{r}_{n} x_{n}\left(1-x_{n}\right)$. We measured the response and analyzed it in terms of the cross correlation function $C_{1}$ between the filtered symbolic output $\sigma_{n}$ and the modulation signal at the input. Figure 10 shows $C_{1}$ as a function of $r$. The result largely coincides with that of the diode resonator. The numerical simulation shows the strongest resonance peak close to the onset of the crisis, $r=r_{c}$, where a small change of the control parameter has a strong effect on the residence times and the jump rates. In accor- dance with the experimental findings smaller resonance peaks are visible as well. Thus, the noise-free SR at an interior crises shows up as a stochastic multiresonance [16,28].

\section{CONCLUSION}

We have proven that noise-free stochastic resonance caused by an interior crisis can be detected in experiments. Although we have adopted a fairly simple electronic circuit the approach does not rely on any peculiar feature of the experimental setup, and it is promising to apply the approach to more intricate time series.

A major constraint for the identification of the resonance phenomenon is caused by the proper identification of the different dynamical phases of the intermittent regime. Since in typical experiments such phases are highly intermingled one cannot apply a simple threshold condition. We have employed here return maps and ideas borrowed from symbolic dynamics for the purpose of state identification. In such a way a quantitative criterion has been developed to separate the different dynamical phases which are related with the precritical attractor and the precritical chaotic repeller, respectively. The approach converts the time series into a symbolic representation which allows the accurate determination of mean residence times and cross correlation functions.

The resonance phenomenon clearly shows up as maxima in the cross correlation function between signal and periodic drive. Apart from the main peak which appears close to the bifurcation point further secondary maxima are visible beyond the crisis. Such kind of multiresonance is caused by the geometric structure of the chaotic attractor. Surprisingly the fine structure observed in the experiment coincides to some degree even quantitatively with the multiresonance observed in the logistic map. It seems to be promising to investigate the generic properties of such a feature.
[1] R. Benzi, A. Sutera, and A. Vulpiani, J. Phys. A 14, 453 (1981).

[2] L. Gammaitoni, P. Hänggi, P. Jung, and F. Marchsoni, Rev. Mod. Phys. 70, 223 (1998).

[3] S. Fauve and F. Heslot, Phys. Lett. 97A, 5 (1983).

[4] S. Barbay, G. Giacomelli, and F. Marin, Phys. Rev. E 61, 157 (2000).

[5] D. F. Russell and F. Moss, Nature (London) 402, 291 (1999).

[6] F. W. Schneider et al., J. Phys. Chem. 100, 4437 (1996); 100, 4442 (1996); 100, 5388 (1996).

[7] M. Diestelhorst and K. Drozhdin, Ferroelectrics 238, 25 (2000).

[8] V. S. Anishchenko, A. B. Neiman, and M. A. Safanova, J. Stat. Phys. 70, 183 (1993).

[9] G. Nicolis, C. Nicolis, and D. McKernan, J. Stat. Phys. 70, 125 (1993)

[10] T. L. Carroll and L. M. Pecora, Phys. Rev. Lett. 70, 576 (1993).

[11] E. Reibold, W. Just, J. Becker, and H. Benner, Phys. Rev. Lett. 78, 3101 (1997).
[12] Ch. Zhou and C. H. Lai, Phys. Rev. E 60, 3928 (1999).

[13] S. L. Ginzburg and M. A. Pustovoit, Europhys. Lett. 45, 540 (1999).

[14] V. M. Chizhevsky, R. Vilaseca, and R. Corbalán, Phys. Rev. E 61, 6500 (2000).

[15] L. Stępień, A. Krawiecki, and R. A. Kosiński, Chaos, Solitons Fractals 19, 1243 (2004).

[16] A. Krawiecki, S. Matyjaskiewicz, K. Kacperski, and J. A. Hołyst, Phys. Rev. E 64, 041104 (2001).

[17] T. Stemler, J. P. Werner, H. Benner, and W. Just, Phys. Rev. Lett. 98, 044102 (2007).

[18] R. Bartussek, P. Hänggi, and P. Jung, Phys. Rev. E 49, 3930 (1994).

[19] P. Saha and S. H. Strogatz, Math. Mag. 68, 42 (1995).

[20] K. G. Szabó, Y.-. Lai, T.Tél, and C. Grebogi, Phys. Rev. E 61, 5019 (2000).

[21] C. Grebogi, E. Ott, F. Romeiras, and J. A. Yorke, Phys. Rev. A 36, 5365 (1987).

[22] C. Grebogi, E. Ott, and J. A. Yorke, Phys. Rev. Lett. 57, 1284 (1986). 
[23] W. L. Ditto, S. Rauseo, R. Cawley, C. Grebogi, G.-H. Hsu, E. Kostelich, E. Ott, H. T. Savage, R. Segnan, M. L. Spano, and J. A. Yorke, Phys. Rev. Lett. 63, 923 (1989).

[24] K. Kacperski and J. A. Hołyst, Phys. Lett. A 254, 53 (1999).

[25] K. Kacperski and J. A. Hołyst, Phys. Rev. E 60, 403 (1999).

[26] C. Grebogi, E. Ott, and J. A. Yorke, Phys. Rev. Lett. 48, 1507
(1982).

[27] B. McNamara and K. Wiesenfeld, Phys. Rev. A 39, 4854 (1989).

[28] J. M. G. Vilar and J. M. Rubí, Phys. Rev. Lett. 78, 2882 (1997). 